\title{
Material Application, Performance and Life Cycle
}

\author{
S Sulaiman, Universiti Putra Malaysia, Serdang, Malaysia
}

(C) 2016 Elsevier Inc. All rights reserved.

\section{Introduction}

The Material Application, Performance and Life Cycle section of Materials Science and Materials Engineering module covers various types of material applications, their performance and life cycle of materials and products. The module itself starts with introducing the properties of metals and nonmetals which include ferrous, nonferrous, ceramics, graphite, diamond, and polymers. Computational modeling and simulation of materials are also included in the introduction of material properties.

For material applications, three main application areas are discussed. These are industrial applications, device applications, and coatings applications. Moreover, it covers corrosion and includes types of corrosion, corrosion in liquid, evaluation, degradation and management, and control of corrosion issues.

For material performance and life cycle, the section discusses fundamentals of material failures. This covers the theories and mechanisms of failure, failure assessment methods, creep and high-temperature failure, environmentally influenced fatigue, interfacial and nanoscale fracture, characterization of failure, and numerical and computational methods.

\section{Material Applications}

Radical materials advances and applications can drive the creation of new products or even new industries, but stable industries also employ materials scientists and engineers to make incremental improvements and troubleshoot issues with currently used materials. Industrial applications play major material applications which include metal and nonmetal. Automotive and aerospace industry are main industrial applications. Industrial applications of materials include materials design, cost-benefit trade-offs in industrial production of materials, processing techniques, and analytical techniques.

Corrosion as a part of product development is also highlighted in this module. It can be defined as the degradation of a material due to a reaction with its environment. It is a natural process, which converts refined metal to their more stable oxide. Degradation implies deterioration of physical properties of the material. Therefore, corrosion management and control become important engineering subject especially in product development and life cycle.

With the increase in computing power, simulating the behavior of materials has become possible. This enables materials scientists and engineers to study properties of materials previously unknown, as well as to design new materials. Up to now, new materials were found by a time consuming trial and error process. But, now it is hoped that computational techniques could drastically reduce that time, and allow us to tailor materials properties. This involves simulating materials at all length scales, using methods such as density functional theory, molecular dynamics, etc.

\section{Materials Performance and Life Cycle}

Materials performance is concerned with degradation phenomena and mechanisms in engineering materials, which determine the life of an engineering component. Life cycle of products depends on the applications and environmental condition. Products in real-life undergo environmental conditions and engineering failure (such as creep and fatigue) that cause gradual degradation to them. Real-life exposure of products involve such varied factors as geometric configuration and details of the construction, composition, porosity, and adherence of corrosion products, environmental pollution, humidity, sun exposure and temperature variations. Besides the choice of material, proper maintenance is another important factor in durability and service life. It has also to be considered that the service life of products is not only a quantifiable technical property, but there is also an aesthetic and fashion input to it, therefore, depends upon economical, functional, social, and physical performance.

Product life cycle management or PLM is another activity which deals with life cycle of products. In industry, product life cycle management is the process of managing the entire life cycle of products from inception, through engineering design and manufacture, to service and disposal of manufactured products. PLM integrates people, data, processes, and business systems, and provides a product information backbone for companies and their extended enterprise. PLM systems help organizations in coping with the increasing complexity and engineering challenges of developing new products for the global competitive markets.

It is planned that a large number of articles (approximately 500) will be published within this very important section dealing with various aspects of application, performances assessment, and life cycle studies of steels, aluminum alloys, composites, polymeric materials, ceramic materials timbers which are finding ever increasing application in all forms of office building and construction industries, aviation industries, domestic housing sectors, and machine parts. 
Articles will also be commissioned and published which will address sources of raw materials, their processing to useable form for construction purposes, service life evaluation, and end of life disposal, and re-cycling prospect.

It is aimed that the assessment of functionality, service life evaluation reported in some of these articles will have been carried out according to the internationally acceptable standards and also some of these test methods may indeed become future standards for some materials for which currently there are no internationally established standards.

The number of articles that have been scheduled to appear in this section of the Reference Module in Materials Science and Materials Engineering which will be launched in December of this year (2015) is a small percentage of the eventual numbers of articles which will be published under this section over the next five years. 